\title{
Additivity of magnetic contributions to the x-ray magnetic circular dichroism spectrum
}

\author{
R. Boada, ${ }^{1,2}$ C. Piquer,,${ }^{1,3}$ M. A. Laguna-Marco, ${ }^{4}$ and J. Chaboy ${ }^{1,2}$ \\ ${ }^{1}$ Instituto de Ciencia de Materiales de Aragón, Consejo Superior de Investigaciones Científicas, CSIC-Universidad de Zaragoza, \\ 50009 Zaragoza, Spain \\ ${ }^{2}$ Departamento de Física de la Materia Condensada, Universidad de Zaragoza, 50009 Zaragoza, Spain \\ ${ }^{3}$ Departamento de Ciencia y Tecnología de Materiales y Fluidos, Universidad de Zaragoza, 50009 Zaragoza, Spain \\ ${ }^{4}$ Advanced Photon Source, Argonne National Laboratory, Argonne, Illinois 60439, USA \\ (Received 4 December 2009; revised manuscript received 11 January 2010; published 5 March 2010)
}

\begin{abstract}
We have studied the behavior of the x-ray magnetic circular dichroism (XMCD) signals recorded at the Fe $K$ edge through the $\mathrm{Ho}_{1-x} \mathrm{Lu}_{x} \mathrm{Fe}_{2}$ series in which the system is driven through a magnetic compensation by varying the concentration of the magnetic rare-earth metal, the temperature and the applied magnetic field. We report a direct experimental evidence of the additive character of the different magnetic species, Ho and Fe, to the XMCD signal at the conduction band. Both magnetic contributions are always present due to the hybridization of the conduction states, and only at $T_{\text {Comp }}$ the Ho contribution to the Fe $K$-edge XMCD disappears.
\end{abstract}

DOI: 10.1103/PhysRevB.81.100404

PACS number(s): 75.50.Bb, 71.20.Lp, 78.20.Ls, 78.70.Dm

The capabilities of x-ray magnetic circular dichroism (XMCD) are nowadays commonly exploited in the study of the localized magnetism of many systems. On the contrary, its application to the study of the conduction-band magnetism is quite scarce, despite the great interest raised by magnetic materials in which the induced magnetic moments in nominally nonmagnetic atoms play an important role into determining their magnetic properties. ${ }^{1-6}$ Consequently, efforts are needed to get a full understanding of the XMCD signals originated at delocalized states. Within this scenario, the study of the $K$ edge of $3 d$ transition metal $(T)$, probing the $4 p$ states, and of the $L$ edges of the rare-earth metals $(R)$, probing the $5 d$ states, provides a unique opportunity to this aim.

Several works have studied the behavior of the XMCD recorded at these absorption edges in the case of $R-T$ intermetallic compounds. It has been demonstrated that the transition metal contributes to the rare-earth $L_{2,3}$-edge XMCD spectra $^{7-9}$ and that there is a contribution of the lanthanide metal to the XMCD recorded at the $K$ edge of the transition metal. ${ }^{10,11}$ In several cases, as the $R \mathrm{Fe}_{2}$ or $R \mathrm{Co}_{2}$ compounds, the rare-earth contribution is as intense as to dominate the overall shape and sign of the XMCD even when one is looking at the transition metal, $\mathrm{Fe}$ and Co $K$ edge. ${ }^{12}$ Although a direct experimental evidence is still missing, previous results suggest that the different magnetic atomic species present in the material contribute additively to the XMCD. ${ }^{7,12}$ As a consequence of this additivity it should be possible to determine the magnetic behavior of the different elements in the material, through the disentanglement of these contributions, by using a single absorption edge. ${ }^{13}$ However, the fact that in several cases the rare-earth contribution dominates the observed XMCD of the transition metal has been interpreted in terms of the rare-earth metal determining not only the shape of the XMCD spectra ${ }^{12}$ but also the magnetism of the transition-metal $4 p$ electrons. ${ }^{14}$ Accordingly, the polarization of the transition-metal $s p$ band should not follow that of the $3 d$ band but it should be determined by the rare-earth magnetization. ${ }^{14}$

In order to get a clear description of both the relationships between the XMCD and the magnetism of the delocalized states, and the origin of the magnetic polarization of these states when the materials contain more than one magnetic species, we have performed the study of the XMCD recorded at the Fe $K$ edge through the $\mathrm{Ho}_{1-x} \mathrm{Lu}_{x} \mathrm{Fe}_{2}$ series. We report a direct experimental evidence of the contributions in additive way of both sublattices, $R$ and $\mathrm{Fe}$, to the XMCD signal at the conduction band.

$\mathrm{Ho}_{1-x} \mathrm{Lu}_{x} \mathrm{Fe}_{2}(x=0,0.25,0.50,0.75$, and 1$)$ samples were prepared by arc melting the pure elements under Ar protective atmosphere. The ingots were annealed at $900{ }^{\circ} \mathrm{C}$ for 1 week. X-ray diffraction characterization indicates that all the samples show a single $C 15$ Laves phase, with the presence of secondary phases being less than $<2 \%$ overall. Magnetization measurements were performed by using a commercial superconducting quantum interference device magnetometer (Quantum Design MPMS-5s). XMCD experiments were performed at the beamline BL39XU of the SPring-8 Facility ${ }^{15}$ on homogeneous layers of the powdered samples. XMCD spectra were recorded in the transmission mode at the Fe $K$ edge by using the helicity-modulation technique. ${ }^{16}$

The Fe $K$-edge XMCD spectra recorded through the $\mathrm{Ho}_{1-x} \mathrm{Lu}_{x} \mathrm{Fe}_{2}$ series at $4.2 \mathrm{~K}$ and $50 \mathrm{kOe}$ are shown in Fig. 1(a). The observed sign reversal between the XMCD signals of $\mathrm{Ho}_{0.25} \mathrm{Lu}_{0.75} \mathrm{Fe}_{2}$ and of those compounds with higher Ho content reflects the change in the dominant magnetic sublattice. It is well known that the coupling between both sublattices, $\mathrm{Ho}$ and $\mathrm{Fe}$, is ferrimagnetic and the magnetization can be described using the two-sublattice model. ${ }^{17}$

When all the XMCD spectra are referred to the direction of the Fe sublattice magnetization one finds [Fig. 1(c)] that the overall shape of the XMCD signal of the compounds containing Ho is markedly different from that of $\mathrm{LuFe}_{2}$. The Fe $K$-edge XMCD spectrum of $\mathrm{LuFe}_{2}$ is similar to that of $\mathrm{Fe}$ metal, showing a main narrow positive peak at the absorption threshold and a negative dip at higher energies. As Ho substitutes $\mathrm{Lu}$ in the $\mathrm{Ho}_{1-x} \mathrm{Lu}_{x} \mathrm{Fe}_{2}$ series the XMCD spectral shape is strongly modified leading to the appearance of spectral features in the high-energy region. The amplitude of the spectra enhances as the Ho content increases in the compound, with the exception of the main positive peak at the threshold which shows the opposite trend. This behavior has 

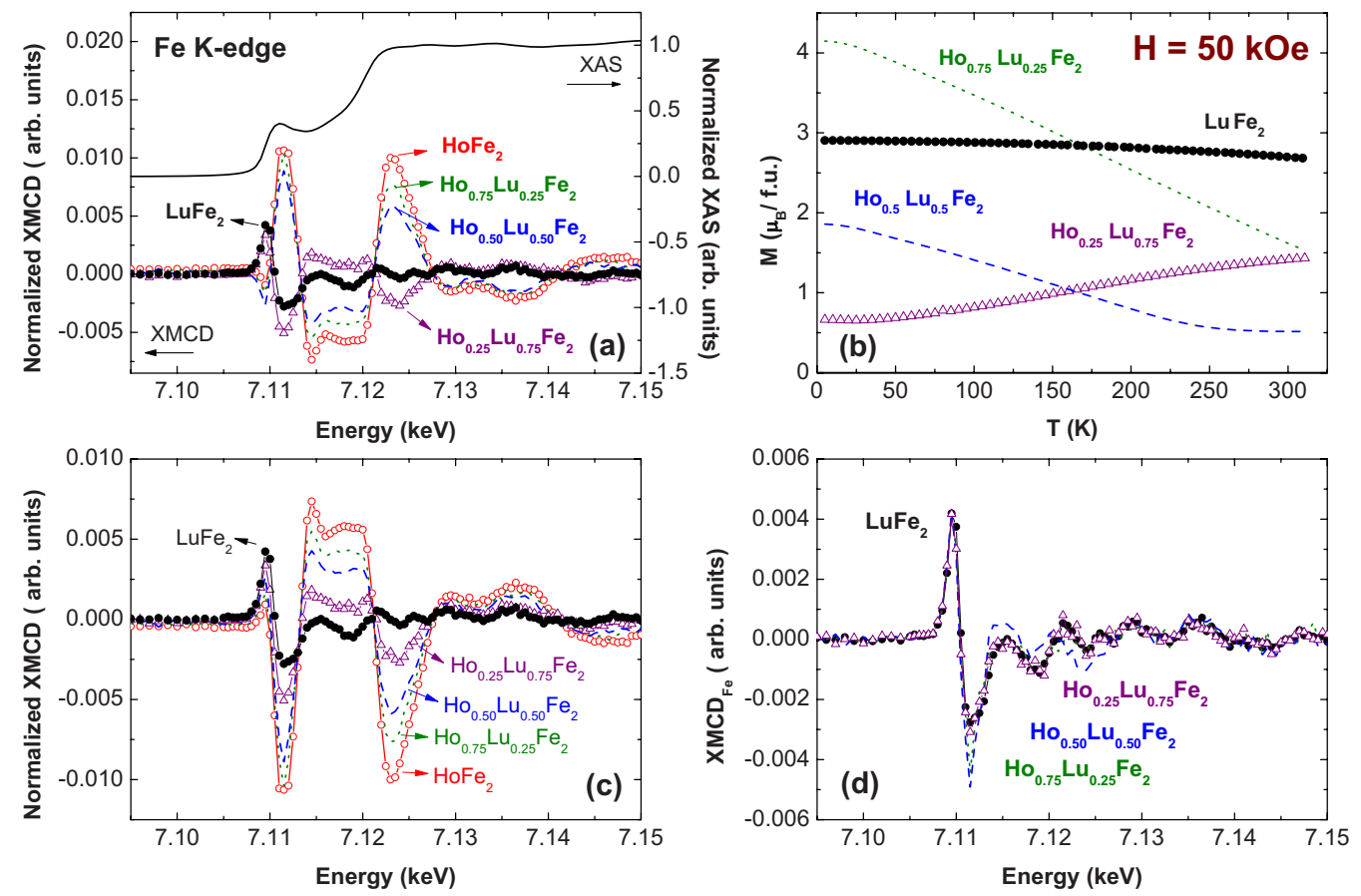

FIG. 1. (Color online) (a) Normalized Fe $K$-edge XMCD spectra recorded at $T=4.2 \mathrm{~K}$ and $H=50$ kOe through the $\mathrm{Ho}_{1-x} \mathrm{Lu}_{x} \mathrm{Fe}_{2}$ series $_{\text {. }}$ (b) Temperature dependence of the magnetization (zero-field cooling) of the $\mathrm{Ho}_{1-x} \mathrm{Lu}_{x} \mathrm{Fe}_{2}$ series measured under an applied magnetic field $H=50 \mathrm{kOe}$. (c) Same as (a) but with the sign of the XMCD signals referred to the direction of the Fe sublattice magnetization. (d) Comparison of the Fe $K$-edge XMCD of $\mathrm{LuFe}_{2}$ and the Fe contribution to the XMCD signal (see text for details). In all the panels: $x=0$ (red $\bigcirc$ ), 0.25 (green dots), 0.5 (blue dashed line), 0.75 (purple $\triangle$ ), and 1 (black $\bigcirc$ ).

been observed in other $R$-Fe intermetallic compounds and accounted for in terms of an additional contribution arising from the magnetic rare-earth sublattice. ${ }^{10-12}$ The question posed is to determine if both Fe and Ho magnetic sublattices contribute in an additive way to the XMCD (Refs. 7 and 11) or, on the contrary, the Ho $4 f$ magnetic moments are which determine the magnetic properties of the $\mathrm{Fe}(s p)$ band. In the former case there should always be a separable contribution from each magnetic element. By contrast, in the latter, the $\mathrm{Fe}(3 d)$ magnetic moments should not play any significant role in the magnetic polarization of the $\mathrm{Fe}(s p)$ band and, consequently, in the Fe $K$-edge XMCD. ${ }^{14}$

Starting from the additivity model, the Fe $K$-edge XMCD spectrum of $\mathrm{HoFe}_{2}$ is composed of an $\mathrm{Fe}$ component, $\mathrm{XMCD}_{\mathrm{Fe}}$, and a contribution from $\mathrm{Ho}, \mathrm{XMCD}_{\mathrm{Ho}}$. According to magnetization measurements the properties of the Fe sublattice remain unvaried through the $R \mathrm{Fe}_{2}$ series. ${ }^{18,19}$ Then, we can assume that the contribution of the Fe sublattice to the $\mathrm{Fe}$ $K$-edge XMCD spectra is the same for both $\mathrm{LuFe}_{2}$ and $\mathrm{HoFe}_{2}$ compounds, and by subtracting both spectra the contribution of the Ho sublattice to the XMCD spectrum of $\mathrm{HoFe}_{2}$ is extracted. In a second step we consider that this contribution has an atomiclike character, i.e., it reflects the polarization of the $5 d$ states due to the localized $4 f$ states of the rare-earth metal which stands from an intra-atomic interaction. If this is the case, the Ho contribution to XMCD through the $\mathrm{Ho}_{1-x} \mathrm{Lu}_{x} \mathrm{Fe}_{2}$ series should be proportional to the extracted signal from $\mathrm{HoFe}_{2}$ weighted by the Ho concentration $\left[(1-x) \times \mathrm{XMCD}_{\mathrm{Ho}}\right]$. By subtracting the scaled Ho contribution from the experimental XMCD spectra we obtain a residual signal that should correspond to $\mathrm{XMCD}_{\mathrm{Fe}}$. If our assumptions are valid, the obtained signal will show the same spectral shape and similar intensity as to resemble the experimental spectrum of $\mathrm{LuFe}_{2}$ in which only the Fe $\mathrm{XMCD}$ contribution should be present as there is no localized $4 f$ moment in the material. The results, reported in Fig. 1(d), show the perfect agreement between both residual and experimental signals, $\mathrm{XMCD}_{\mathrm{Fe}}$ and $\mathrm{LuFe}_{2}$, respectively, giving validity to our hypothesis.

The next step is to verify if the successful application of the additivity model is limited to the present experimental conditions, $T=4.2 \mathrm{~K}$ and $H=50 \mathrm{kOe}$, in which all the magnetic moments are close to their saturation values, or if it can be extended through all the accessible range of temperatures and applied magnetic fields. Consequently, we have recorded the XMCD signals at $T=300 \mathrm{~K}$ under an applied magnetic field of $H=20 \mathrm{kOe}$. Moreover, this coincides with the magnetic compensation condition for the $\mathrm{Ho}_{0.5} \mathrm{Lu}_{0.5} \mathrm{Fe}_{2}$ compound. It is worth to note that the Neel temperatures $T_{N}$ of these compounds are around $600 \mathrm{~K}\left(\mathrm{LuFe}_{2}: 582 \mathrm{~K}, \mathrm{HoFe}_{2}\right.$ : $606 \mathrm{~K}$, and $T_{N}$ of the diluted compounds follows a linear variation between the parent compounds). The results of the XMCD measurements are shown in Fig. 2(a) (for the sake of simplicity they have been plotted referred to the direction of the Fe sublattice magnetization). This comparison shows that the spectral shape of the signals for the Ho compounds, except $x=0.5$, is the same as that at low temperature, and that only their amplitude has been modified. This result gives further support to the atomiclike nature of the Ho contribution to the XMCD of Fe. In the case of $\mathrm{Ho}_{0.5} \mathrm{Lu}_{0.5} \mathrm{Fe}_{2}$ the compensation condition has been reached, $T_{\text {Comp }} \sim 300 \mathrm{~K}$, and the Fe $K$-edge XMCD spectrum has lost all the hall- 

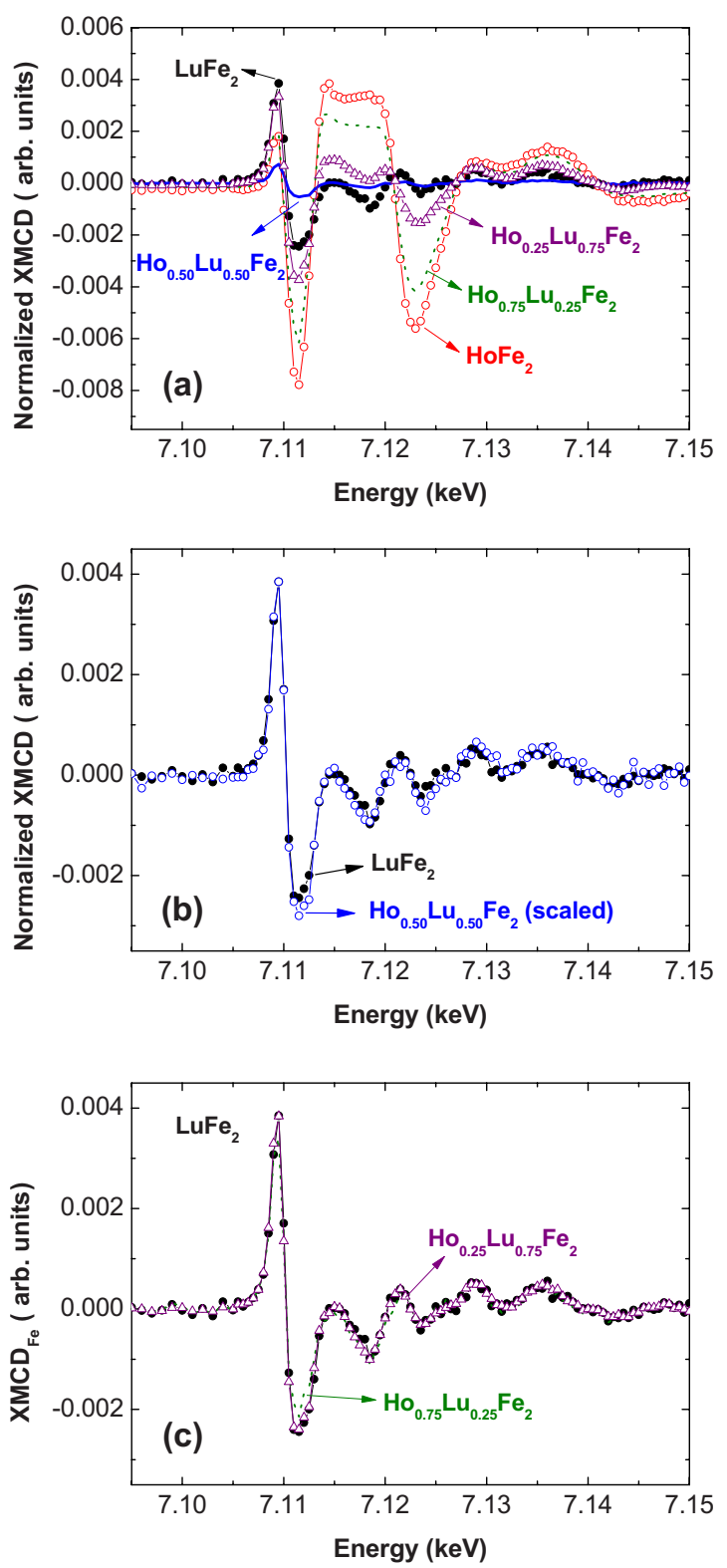

FIG. 2. (Color online) (a) Normalized Fe $K$-edge XMCD spectra recorded at $T_{\text {Comp }}$ and $H=20 \mathrm{kOe}$ through the $\mathrm{Ho}_{1-x} \mathrm{Lu}_{x} \mathrm{Fe}_{2}$ series: $x=0$ (red $\bigcirc$ ), 0.25 (green dots), 0.5 (blue line), 0.75 (purple $\triangle$ ), and 1 (black ). (b) Detailed comparison of the XMCD signal recorded for $\mathrm{LuFe}_{2}$ (black - ) and $\mathrm{Ho}_{0.5} \mathrm{Lu}_{0.5} \mathrm{Fe}_{2}$ (blue $\bigcirc$ ). (c) Comparison of $\mathrm{LuFe}_{2} \mathrm{XMCD}$ (black - and the extracted $\mathrm{XMCD}_{\mathrm{Fe}}$ signals of $x=0.25$ (green dots) and 0.75 (purple $\triangle$ ).

marks of this Ho contribution. Indeed, as shown in Fig. 2(b), the XMCD spectrum perfectly matches with that of $\mathrm{LuFe}_{2}$, i.e., with the compound in which no $4 f$ localized moments are present. The spectra of the $\mathrm{Ho}_{0.5} \mathrm{Lu}_{0.5} \mathrm{Fe}_{2}$ compound have been scaled with the $\mathrm{LuFe}_{2}$ ones at the main peak of the XMCD signal $(7109.5 \mathrm{eV})$. The scaling factor needed coincides with the ratio of the magnetization value of both $\mathrm{LuFe}_{2}$ and $\mathrm{Ho}_{0.5} \mathrm{Lu}_{0.5} \mathrm{Fe}_{2}$ at the same experimental conditions.

This result can be addressed to the absence of a net Ho magnetization in the direction of the magnetic applied field at the compensation temperature. However, when the system is taken out of the compensation condition a net magnetiza-

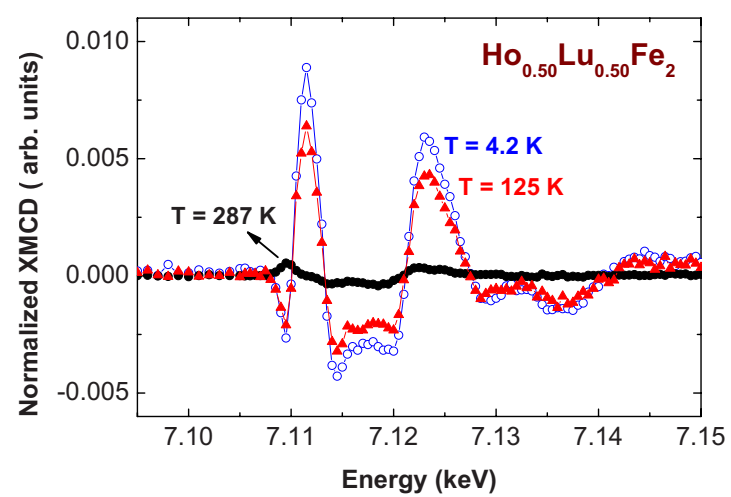

FIG. 3. (Color online) Comparison of the Fe $K$-edge XMCD spectra of $\mathrm{Ho}_{0.5} \mathrm{Lu}_{0.5} \mathrm{Fe}_{2}$ recorded at $H=50 \mathrm{kOe}$ and different temperatures: $T=287$ (black $\bigcirc$ ), 125 (red $\mathbf{\Delta}$ ), and 4.2 (blue $\bigcirc$ ) $\mathrm{K}$.

tion appears at the Ho sublattice. As a result, the magnetic coupling between both sublattices is recovered and the XMCD signal at the Fe $K$ edge shows the polarization of the $\mathrm{Fe}(4 p)$ states due to the Ho atoms. It is shown in Fig. 3 that at temperatures relatively far from the compensation, $T=125 \mathrm{~K}$, the spectral shape of the whole Ho-containing compounds is recovered.

In summary, we have studied the behavior of the $\mathrm{Fe}$ $K$-edge XMCD through the magnetic compensation occurring in the $\mathrm{Ho}_{1-x} \mathrm{Lu}_{x} \mathrm{Fe}_{2}$ series. Our results prove that both $\mathrm{Ho}$ and Fe sublattices contribute to the XMCD of Fe in an additive way. Only at $T_{\text {Comp }}$, when the net Ho sublattice magnetization is canceled, the Ho contribution to the Fe $K$-edge $\mathrm{XMCD}$ disappears and the spectrum becomes similar to that of $\mathrm{LuFe}_{2}$, i.e., a system in which no localized $4 f$ moment is present. These results show that the rare-earth contribution to the $K$-edge XMCD of the transition metal reflects the net magnetization of the rare-earth metal and can be considered as of atomiclike nature, i.e., the rare-earth $5 d$ states become spin polarized by the intra-atomic interaction with the $4 f$ localized moments and the spin polarization of the $5 d$ states is probed in the photoabsorption process due to the $R-T$ hybridization. The fact that it arises from the localized $4 f$ states explains why for a fixed rare-earth metal in a $R-T$ series the shape of the observed contribution to the $K$-edge XMCD of the transition metal is similar independent of the specific transition metal. ${ }^{12,18}$ Moreover, even when in several cases the rare-earth contribution dominates the observed XMCD, this work proves that the $\mathrm{Fe}$ intra-atomic $3 d$ polarization is always present at the $\mathrm{Fe}(s p)$ band. This polarization is only intrinsic to the $\mathrm{Fe}$ sublattice and it remains throughout the whole temperature range, even at the magnetic compensation point.

This work was partially supported by Spanish CICYT Grant No. MAT2008-06542-C04-01. The synchrotron radiation experiments were performed at SPring- 8 within the Budding Researchers Support Program (Proposal No. 2008B1753). We are indebted to M. Suzuki and N. Kawamura for the experimental support at SPring-8. M.A.L.-M. and R.B. acknowledge support from the Ministerio de Ciencia e Innovación of Spain. 
${ }^{1}$ D. Haskel, Y. B. Lee, B. N. Harmon, Z. Islam, J. C. Lang, G. Srajer, Y. Mudryk, K. A. Gschneidner, and V. K. Pecharsky, Phys. Rev. Lett. 98, 247205 (2007).

${ }^{2}$ N. M. Souza-Neto, D. Haskel, J. C. Lang, O. Chmaissem, B. Dabrowski, and I. Felner, Phys. Rev. B 80, 140414(R) (2009).

${ }^{3}$ J. Chaboy, P. Lázpita, J. M. Barandiarán, J. Gutiérrez, M. L. Fernández-Gubieda, and N. Kawamura, J. Phys.: Condens. Matter 21, 016002 (2009).

${ }^{4}$ N. Jaouen, F. Wilhelm, A. Rogalev, J. Goulon, L. Ortega, J. M. Tonnerre, and A. Yaresko, J. Phys.: Condens. Matter 20, 095005 (2008).

${ }^{5}$ J. Chaboy, F. Bartolomé, M. R. Ibarra, C. I. Marquina, P. A. Algarabel, A. Rogalev, and C. Neumman, Phys. Rev. B 59, 3306 (1999).

${ }^{6}$ A. García Prieto, M. L. Fdez-Gubieda, J. Chaboy, M. A. LagunaMarco, T. Muro, and T. Nakamura, Phys. Rev. B 72, 212403 (2005).

${ }^{7}$ M. A. Laguna-Marco, J. Chaboy, and H. Maruyama, Phys. Rev. B 72, 094408 (2005).

${ }^{8}$ M. A. Laguna-Marco, J. Chaboy, C. Piquer, H. Maruyama, N. Ishimatsu, N. Kawamura, M. Takagaki, and M. Suzuki, Phys. Rev. B 72, 052412 (2005).

${ }^{9}$ M. A. Laguna-Marco, J. Chaboy, and C. Piquer, Phys. Rev. B 77, 125132 (2008).
${ }^{10}$ J. Chaboy, H. Maruyama, L. M. García, J. Bartolomé, K. Kobayashi, N. Kawamura, A. Marcelli, and L. Bozukov, Phys. Rev. B 54, R15637 (1996).

${ }^{11}$ J. Chaboy, M. A. Laguna-Marco, M. C. Sánchez, H. Maruyama, N. Kawamura, and M. Suzuki, Phys. Rev. B 69, 134421 (2004).

${ }^{12}$ M. A. Laguna-Marco, C. Piquer, and J. Chaboy, Phys. Rev. B 80, 144419 (2009).

${ }^{13}$ J. Chaboy, M. A. Laguna-Marco, C. Piquer, R. Boada, H. Maruyama, and N. Kawamura, J. Synchrotron Radiat. 15, 440 (2008).

${ }^{14}$ J. Herrero-Albillos, D. Paudyal, F. Bartolomé, L. M. García, V. K. Pecharsky, J. K. A. Gschneider, A. T. Young, N. Jaouen, and A. Rogalev, J. Appl. Phys. 103, 07E146 (2008).

${ }^{15}$ H. Maruyama, J. Synchrotron Radiat. 8, 125 (2001).

${ }^{16}$ M. Suzuki, N. Kawamura, M. Mizumaki, A. Urata, H. Maruyama, S. Goto, and T. Ishikawa, Jpn. J. Appl. Phys., Part 2 37, L1488 (1998).

${ }^{17}$ K. H. J. Buschow and F. R. de Boer, Physics of Magnetism and Magnetic Materials (Kluwer Academic Publishers, New York, 2003).

${ }^{18}$ M. A. Laguna-Marco, A New Insight into the Interpretation of the $T K$-Edge and $R L_{2,3}$-Edges XMCD Spectra in R-T Intermetallics (Prensas Universitarias de Zaragoza, Zaragoza, 2007).

${ }^{19}$ E. Burzo, Rep. Prog. Phys. 61, 1099 (1998). 